Rev. salud pública. 14 sup (2): 15-31, 2012

\title{
Defunciones en niños a principios de los siglos XX y XXI en Boyacá-Colombia
}

\section{Deaths in children during the early twentieth and twenty-first century in Boyacá, Colombia}

\author{
Fred G. Manrique-Abril 1,3, Abel F. Martínez-Martín ${ }^{2}$ y \\ Bernardo F.Meléndez-Álvarez ${ }^{2}$
}

1 Escuela de Enfermería, Universidad Pedagógica y Tecnológica de Colombia. Tunja, Colombia. fred
.manrique@uptc.edu.co
2 Escuela de Medicina, Universidad Pedagógica y Tecnológica de Colombia. Tunja, Colombia. abelfma
rtinez@gmail.com; bfmelendez@ gmail.com
3 Facultad de Enfermería, Universidad Nacional de Colombia. Bogotá, Colombia. fgmanriquea@unal. edu.co
Recibido 25 Mayo 2011/Enviado para Modificación 8 Agosto 2011/Aceptado 3 Noviembre 2011

RESUMEN

Objetivo Comparar y analizar las Tasas Brutas de Mortalidad (TBM), la Mortalidad en Menores (MN) de 5 años por mil Nacidos Vivos (NV) y la Mortalidad Infantil (MI) en el departamento de Boyacá-Colombia, a comienzos de los siglos XX y XXI.

Métodos Estudio descriptivo comparativo con enfoque histórico epidemiológico. Se utilizaron dos fuentes de datos, para las defunciones: registros parroquiales de 1912-1927 y el Boletín epidemiológico de Boyacá 2007; para la población los censos DANE 1912-1918-1927 y 2005. Se almacenaron y analizaron en Mysql囚. Se calcularon tasas brutas, específicas por edad e infantil, proporciones de mortalidad y mortalidad por causas.

Resultados Entre 1912-1927 se registra una media de 7958 muertes, en comparación con 5813 en el siglo XXI; la TBM pasó de 150 muertes a 42 por 10 mil habitantes; la TMI se redujo considerablemente pasando de 231 a 17 por cada $1000 \mathrm{NV}$; aunque se pueden comparar las tasas especificas en menores de 8 años con las de menores de 5 , existe variabilidad en el rango y en la construcción de la TM especifica y la definida por OMS- UNICEF.

Conclusiones Existe un impacto en la reducción de mortalidad, mayor en el siglo $\mathrm{XXI}$, debido a las políticas nacionales e internacionales de control de enfermedades inmunoprevenibles y muerte materna. La pandemia de gripa de 1918-19 afectó considerablemente la mortalidad en todos los grupos de población; las causas violentas ocupan lugares importantes en la mortalidad infantil en el siglo XXI.

Palabras Clave: Tasa de mortalidad, tasa de mortalidad infantil, mortalidad en la infancia, registros de mortalidad, historia (fuente: DeCS, BIREME). 


\section{ABSTRACT}

Objective Comparing and analysing crude death (CDR), child mortality (CMR) 5 -year per thousand live births (LBR) and infant mortality rates (IMR) in the Boyacá department, Colombia, during the early $20^{\text {th }}$ and $21^{\text {st }}$ centuries.

Methods A descriptive epidemiological comparative historical approach was adopted. Two data sources were used for deaths: parish registers 1912-1927 and the Boyacá Epidemiological Bulletin 2007 population census for 1912-1918-1927 and 2005 (Colombian Statistics Bureau - DANE); data was stored and analysed in Mysql. Crude rates, age-specific and child mortality ratios and mortality were calculated by cause.

Results 7,958 deaths were recorded from 1912-1927 compared to 5,813 in the $21^{\text {st }}$ century. CDR dropped from 150 to 42 deaths per 10,000 inhabitants; the IMR became significantly reduced from 231 to 17 for every 1,000 LB. Although specific rates for children under 8 years of age could be compared to children under 5 , there was variability in the range and construction of the specific MR and that defined by WHO-UNICEF.

Conclusions There was a higher reduction in mortality in the $21^{\text {st }}$ century due to national and international policies for controlling preventable diseases and maternal death. The 1918-19 flu pandemic significantly affected mortality in all population groups; violent causes were prominent in infant mortality in the $21^{\text {st }}$ century.

Key Words: Mortality rate, infant mortality rate, child mortality, mortality registry, history (source: MeSH, NLM).

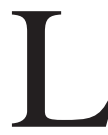

os registros de defunción realizados en el pasado y su comparación con los indicadores de mortalidad del presente, a pesar de las dificultades siglo XX como los sistematizados por los profesionales en informática en el siglo XXI, permiten analizar el comportamiento del proceso salud enfermedad, proponiendo, con visión histórica, direccionar políticas en mejora de la calidad de vida y su esperanza para el futuro.

A través de la historia, los indicadores demográficos y de salud han cambiado sustancialmente a causa de las políticas, las prioridades gubernamentales de intervención en la población y a diversos intereses, modificando a través del tiempo la manera de medir la estructura poblacional, registrándose cambios en la nominación de las causas de muerte, dividiendo la población en diversos rangos de edad, cambiando conceptualmente la denominación de las etapas del proceso vital así como la construcción estadística de los indicadores. Si bien, la mortalidad está lejos de ser una medida ideal del estado de salud de una 
población, es uno de los indicadores, sino el único, al que se tiene fácil acceso. La medida más simple de mortalidad es la Tasa Bruta de Defunciones (TBD), construida como la relación entre el total de defunciones sobre la población por mil, en un lugar y periodo determinados (1). Los indicadores de mortalidad permiten evidenciar la efectividad y capacidad de respuesta de las autoridades sanitarias nacionales e internacionales. A finales del siglo XX, la OMS consideró como prioridad reducir la mortalidad en los niños y las maternas para lograr el objetivo de "Salud para Todos en el año 2000", mediante la implementación de estrategias como el Programa Ampliado de Inmunizaciones (PAI) creado en 1974, que buscó la prevención de las enfermedades transmisibles en la población infantil, a través del uso de vacunas (2).

Además, los programas de salud materno-infantiles en los países latinoamericanos han producido un notable impacto positivo, con descenso de las tasas de mortalidad y disminución de algunas tasas de morbilidad, especialmente de las enfermedades infecciosas transmisibles en general e inmunoprevenibles en particular.

En el período 1960-1990, la mortalidad infantil en las regiones en desarrollo se redujo a la mitad, es decir, una defunción por cada 10 niños menores de cinco años. La MI es inversamente proporcional al desarrollo de un país y permite comparar la situación de un país o región a través de la historia. Ha sido tradicionalmente considerada uno de los indicadores más sensibles de la situación de salud de una comunidad y también un buen marcador del nivel de vida de las poblaciones (3).

La OMS considera muertes preescolares a las ocurridas en niños de 1 a 5 años; muertes infantiles a las ocurridas en menores de 1 año. El mejor indicador para medir la exposición acumulada al riesgo de morir durante los primeros años de vida es la mortalidad en la infancia, que abarca desde el momento del nacimiento hasta antes de los cinco años de edad. La UNICEF y la OMS definieron como indicador de seguimiento de los Objetivos de Desarrollo del Milenio (ODM) el uso de la probabilidad de morir antes de los cinco años, la cual se obtiene al dividir las muertes de menores de cinco años de un lugar y período determinado sobre los nacidos vivos (NV) de ese mismo lugar y período, para después multiplicar el cociente por 1000 , reconocida como Tasa de Mortalidad en Menores de 5 años por 1000 nacidos vivos (4). 
Demógrafos y epidemiólogos recomiendan el cálculo de la Tasa de Mortalidad Especifica (TME), que para la población menor de 5 años sería igual a las defunciones en el grupo, en un periodo y lugar determinado, dividido entre la población menor de 5 años en el mismo periodo y lugar, para después multiplicar el cociente por 1000. Este indicador es poco usado en las estadísticas oficiales.

La Tasa de Mortalidad Infantil (TMI), se refiere a las muertes ocurridas durante el primer año de vida y se expresa como tasa por mil nacidos vivos (NV) (5). La OMS, en los ODM estableció como prioridad disminuir la mortalidad infantil ya que cada año mueren más de 9 millones de niños menores de 5 años. El $90 \%$ de estas defunciones se deben a seis causas: problemas neonatales, neumonía, diarrea, paludismo, sarampión y VIH/SIDA (6). El peso que tiene la mortalidad en un grupo etéreo frente a la mortalidad general se expresa en la Proporción de Mortalidad (PM) (7).

Boyacá con $8630 \mathrm{Km}^{2}$ de extensión, registra 503000 habitantes en 1905 y 586000 en 1912. El departamento perdió importancia nacional pasando en 1912 al quinto puesto en población, con una inserción prácticamente inexistente en la economía mundial (8); lo mismo puede decirse de la modernización cultural (1): "El pueblo boyacense permanece dentro de sus serranías y sus planicies como extraño al movimiento de civilización que hoy conmueve al país (...) La higiene es completamente desconocida.” (9). En las primeras décadas del siglo XX, Boyacá sigue siendo un departamento rural, aislado del país y del exterior, sin desarrollo industrial, manejado por los hacendados, el clero y el Partido Conservador. El periódico liberal La linterna, escribe en 1918: “...enclavado Boyacá en el corazón del país (...) le ha tocado vegetar hasta hoy agobiado entre una deprimente miseria. Lo que su suelo produce está obligado a consumirlo casi del todo, pues sin caminos, sin una vía fluvial aprovechable, sin una cuarta de riel, apenas si tiene un intercambio comercial rudimentario..." (10).

En 1916 funcionaban hospitales, con sus respectivas juntas de beneficencia en 25 municipios del departamento de Boyacá. El gobernador en 1919, afirma que estos "Se sostienen de la caridad Pública y, en especial, debido al celo y trabajo de los señores Curas Párrocos y a la Abnegación y heroicas virtudes de las nobles Hermanas de la Caridad". Todavía en la década de los 30 del siglo XX: "Los prestigiosos doctores tunjanos Aquileo Espinosa, Juan Clímaco Hernández y Silvino Rodríguez, solo disponían de una aguja para inyecciones hipodérmicas y tenían que prestársela mutuamente cuando la necesitaban"(10). 
Desde la reforma de la constitución en 1991 surgieron cambios importantes para la atención en salud, lo primero fue al expedición de la ley 100 de 1993 que buscaba la cobertura universal de servicio de salud para todos los colombianos a través de dos regímenes, el contributivo y el subsidiado (7).

Se incluyó un Plan de Atención Básica que permitiría reducir los indicadores de mortalidad mediante estrategias de Atención primaria en salud y la continuidad y monitoreo del Programa Ampliado de inmunizaciones, Vigilancia epidemiológica y reducción de la mortalidad materna entre otros programas.

El censo de 2005 registro para Boyacá 1255311 habitantes y se reportaron $632836(52 \%)$ en las cabeceras municipales, volviéndose de predominio urbano por primera vez en la historia (11). El objetivo de este artículo es comparar y analizar las Tasas Brutas de Mortalidad, la Mortalidad en Menores de 5 años por mil NV y la Mortalidad Infantil en el departamento de BoyacáColombia, a comienzos de los siglos XX y XXI.

\section{METODOLOGIA}

Se realizó un estudio descriptivo comparativo con enfoque histórico epidemiológico. Se utilizaron dos fuentes de datos, para comienzo del siglo $\mathrm{XX}$, la base de datos del proyecto de investigación: "Análisis histórico epidemiológico de la Pandemia de Gripa de 1918-19 en Boyacá"(12-14) en el que se recolectaron registros de defunción parroquiales de los años 1912 a 1927 en 82 municipios en Boyacá. Se incluyeron 127322 registros de mortalidad general, 53514 de mortalidad en menores de 8 años, de los cuales 31948 corresponden a menores de un año. Para el periodo 2000 a 2005, se analizaron los datos del censo DANE 2005 (11), la Encuesta Nacional de Salud 2007(15) y los boletines epidemiológicos del departamento de Boyacá 2007-2008, entre varias fuentes consultadas que se descartaron por las inconsistencias en las cifras y en los cálculos de los indicadores.

Además, se revisaron fuentes documentales de la época, informes del gobernador de Boyacá a la Asamblea Departamental y la Revista Médica de Bogotá, órgano oficial de la Academia Nacional de Medicina para los inicios del siglo XX. Se proyectó la población para los años 1912 a 1927 con los censos de 1912, 1918 y 1928. La población del periodo 2000-2005 se extrajo de las proyecciones a partir de los censos de 1993 y 2005 (11). 
Se calcularon las tasas brutas de mortalidad, la tasa de mortalidad infantil, las tasas específicas de mortalidad con la mortalidad en menores de 8 años y en menores de 5 años y, por último, se calcularon las proporciones de mortalidad para menores de 1 año, menores de 5 años o de 8 años, según el periodo histórico y la disponibilidad de los datos.

La base poblacional para el cálculo de las tasas de mortalidad infantil para comienzos del siglo XX fue la población menor de un año, mientras que la base poblacional para el siglo XXI fueron los nacidos vivos. Así mismo la base poblacional para la tasa de mortalidad específica en menor de 8 y 5 fue la población en ese mismo rango. Además se calculó la mortalidad en menores de 8 y 5 años por mil NV ajustando el estudio a los criterios de la OMS y UNICEF para el seguimiento de los ODM (6).

Para el análisis por causas de mortalidad infantil y menores de 8 años, se agruparon los diagnósticos en 26 categorías, en los registros parroquiales y se reagruparon las 10 primeras causas del reporte epidemiológico del año 2005. Se aclara que los censos de comienzo de siglo XX contaban la población general pero en la estratificación por sexo y edades no se registraban las mujeres, solo se consideran en el registro a los hombres. En los censos de 1912, 1918 y 1928 los grupos poblacionales se reportaban en intervalos diferentes a los actuales, el grupo que ha permanecido uniforme es el de menores de un año, el hoy llamado por la OMS grupo preescolar que va de 1 a 4 años de edad en aquella época era reportado de 1 a 7 años y se denominaban párvulos. La información se almacenó y analizó en una base de datos en MYSQL®.

Mortalidad general

Los datos de la Tabla 1 presentan el número habitantes y las defunciones registradas en 82 municipios de Boyacá a comienzos del siglo XX; junto a la población y la mortalidad general de 123 municipios, según censo de 2005 para comienzos del siglo XXI. En el periodo 1912-27 la tasa bruta de mortalidad bajo de 175,1 a 116,5 defunciones por 10000 habitantes, pero se observa que hay incrementos en 1915-16 y en 1918 año en el que ocurre la peor pandemia de la historia, la pandemia de gripa española.

Si observamos la tasa bruta de mortalidad durante el periodo 2000-2005 no hay grandes cambios, manteniéndose una tendencia de 42 muertes por cada 10 mil habitantes en Boyacá. La tasa bruta de mortalidad comparada con 
la registrada a comienzo del siglo $\mathrm{XX}$ se redujo significativamente frente a 1927, aunque en valores absolutos el número de defunciones se mantienen constantes en 93 años, observándose el efecto de la dilución poblacional. Las cifras absolutas de mortalidad en 2005 son similares a las registradas 80 años antes, en 1925.

Tabla 1. Mortalidad general en Boyacá

\begin{tabular}{cccc}
\hline Año & $\begin{array}{c}\text { Población } \\
\text { Total }\end{array}$ & $\begin{array}{c}\text { Defunciones } \\
\text { totales }\end{array}$ & $\begin{array}{c}\text { TBM } \times 10 \text { mil } \\
\text { habitantes }\end{array}$ \\
\hline 1912 & 438726 & 7681 & 175,1 \\
1913 & 447346 & 6752 & 150,9 \\
1914 & 457069 & 7754 & 169,6 \\
1915 & 467924 & 9885 & 211,3 \\
1916 & 480019 & 9896 & 206,2 \\
1917 & 493481 & 7887 & 159,8 \\
1918 & 502389 & 9600 & 191,1 \\
1919 & 520963 & 7714 & 148,1 \\
1920 & 539859 & 8314 & 154,0 \\
1921 & 560032 & 7871 & 140,5 \\
1922 & 580596 & 7892 & 135,9 \\
1923 & 601683 & 7302 & 121,4 \\
1924 & 623250 & 6239 & 100,1 \\
1925 & 646100 & 6505 & 100,7 \\
1926 & 669793 & 7884 & 117,7 \\
1927 & 699361 & 8146 & 116,5 \\
2000 & 1365669 & 5828 & 42,7 \\
2001 & 1375222 & 5465 & 39,7 \\
2002 & 1385184 & 5881 & 42,5 \\
2003 & 1394952 & 5868 & 42,1 \\
2004 & 1404309 & 5778 & 41,1 \\
2005 & 1413064 & 6058 & 42,9 \\
\hline TBM: Tasa Bruta de Mortalidad &
\end{tabular}

No se analizan ni presentan las causas de mortalidad general ya que en el registro 1912-1927 se pueden contabilizar más de 1000 diagnósticos posibles y su recodificación será fruto de futuros trabajos que no son el objetivo del presente artículo.

Mortalidad en menor de 8 años y menor de 5 años

En la Tabla 2 se observa una disminución de la tasa de mortalidad específica en menores de 8 años desde el año 1913 hasta 1927. Al igual que en la tasa bruta de mortalidad, la tasa de mortalidad especifica en menores de 8 años se ve incrementada en los años 1915, 1916 y 1918. 
En el Siglo XXI la mortalidad en menores de 5 años disminuyó tanto en valores absolutos como en la tasa especifica. El promedio de defunciones en el periodo 2000-2005 se redujo en un $86 \%$ respecto al promedio de defunciones registrado en el periodo 1912-1927. La tasa promedio de mortalidad específica en menores de 5 años, se redujo en un 94,6 \% entre comienzos del siglo XX y XXI, considerando la limitación en los intervalos poblacionales de los dos rangos comparados.

Tabla 2. Mortalidad en menores de 8, 5 y 1 año en Boyacá

\begin{tabular}{cccccccc}
\hline Año & $\begin{array}{c}\text { Población } \\
<8 \text { años }\end{array}$ & $\begin{array}{c}\text { Defun- } \\
\text { ciones } \\
<8 \text { años }\end{array}$ & $\begin{array}{c}\text { TME } \\
\text { X } 1 \text { 000 } \\
<8 \text { años }\end{array}$ & $\begin{array}{c}\text { Mortalidad } \\
\text { en<8 años X } \\
1000 \text { NV }\end{array}$ & $\begin{array}{c}\text { Población } \\
<1 \text { año }\end{array}$ & $\begin{array}{c}\text { Defunciones } \\
<1 \text { año }\end{array}$ & $\begin{array}{c}\text { TMl } \\
\text { X } 1000 \\
\text { NV }\end{array}$ \\
\hline 1912 & 23104 & 2827 & 122,4 & 785,28 & 3600 & 1819 & 505,3 \\
1913 & 50147 & 2932 & 58,5 & 374,53 & 7829 & 1922 & 245,5 \\
1914 & 51237 & 3416 & 66,7 & 427,07 & 7999 & 1901 & 237,7 \\
1915 & 52454 & 4373 & 83,4 & 534,03 & 8189 & 2278 & 278,2 \\
1916 & 53810 & 4121 & 76,6 & 490,58 & 8400 & 2149 & 255,8 \\
1917 & 55319 & 2886 & 52,2 & 334,19 & 8636 & 1787 & 206,9 \\
1918 & 56318 & 3735 & 66,3 & 424,83 & 8792 & 2327 & 264,7 \\
1919 & 58400 & 3380 & 57,9 & 370,74 & 9117 & 2162 & 237,1 \\
1920 & 60518 & 3427 & 56,6 & 362,74 & 9448 & 2089 & 221,1 \\
1921 & 62780 & 3502 & 55,8 & 357,33 & 9801 & 2407 & 245,6 \\
1922 & 65085 & 3318 & 51,0 & 326,56 & 10160 & 1735 & 170,8 \\
1923 & 67449 & 3064 & 45,4 & 290,99 & 10529 & 1923 & 182,6 \\
1924 & 69866 & 2265 & 32,4 & 207,67 & 10907 & 1324 & 121,4 \\
1925 & 72428 & 2725 & 37,6 & 241,01 & 11307 & 1807 & 159,8 \\
1926 & 75084 & 3700 & 49,3 & 315,66 & 11721 & 2207 & 188,3 \\
1927 & 78398 & 3843 & 49,0 & 314,00 & 12239 & 2111 & 172,5 \\
2000 & 158757 & 518 & 3,3 & 23,15 & 22379 & 414 & 18,5 \\
2001 & 157560 & 471 & 3,0 & 23,08 & 20404 & 383 & 18,8 \\
2002 & 156406 & 486 & 3,1 & 21,65 & 22450 & 401 & 17,9 \\
2003 & 152341 & 420 & 2,8 & 19,88 & 21132 & 339 & 16,0 \\
2004 & 153672 & 426 & 2,8 & 20,28 & 21006 & 337 & 16,0 \\
2005 & 145298 & 449 & 3,1 & 21,88 & 20523 & 357 & 17,4 \\
\hline Nota:<5 años: menor de 5 años, <8 años: menores de 8 años; NV: nacidos vivos; TME: tasa de mortalidad \\
especifica; Mortalidad en <8 < <5: probabilidad de muerte a los 8 o 5 años con relación a los NV.
\end{tabular}

Al aplicar el indicador de la OMS y UNICEF en la evaluación de mortalidad en la infancia en menores de 5 años y 8 años con relación a NV se pueden observar los resultados en última columna de la Tabla 2; se aprecia que la constante es muy similar a la tasa de mortalidad especifica calculada para el grupo poblacional de menores de 8 y menores de 5 años. Este indicador permite evidenciar con detalle el impacto en la reducción de la mortalidad y el mejoramiento de la supervivencia hasta los 5 años. 
En la Tabla 3, las causas curiosas, desconocidas, mal definidas o sin dato, no se ordenan dentro las causas de mortalidad ya que no representan una causa específica y clara de mortalidad, reflejando el inadecuado registro hecho por los curas párrocos de la época. De la misma forma las causas mal definidas persisten como causa reportada en el 2005.

Dentro de las causas curiosas se destaca la muerte durante el bautizo con 106 registros y 2 de ellos muy específicos como "ahogamiento en la pila bautismal" tal vez fruto de la inmersión en la pila; sobresalen otras causas como "El Bastardo", que no se refiere a la legitimidad de la paternidad del menor con 129 casos; por último aparecen registradas 22 casos como "tocado de difunto" causa que persiste en la mortalidad sentida en las zonas rurales de Boyacá. La principal causa de mortalidad en menores de 8 años, que se evidencio a comienzo del siglo XX, fue la enfermedad respiratoria sin incluir la gripa, la cual representó un $32 \%$ del total de la mortalidad del periodo analizado.

Tabla 3. Causas de Mortalidad en menores de 8 años-Boyacá, 1912-1927 Números absolutos

\begin{tabular}{|c|c|c|c|c|c|c|c|c|c|}
\hline Causa/año & $1912-13$ & 1914-15 & $1916-17$ & 1918-19 & $1920-21$ & $1922-23$ & $1924-25$ & $1926-27$ & Total \\
\hline $\begin{array}{l}\text { Enf } \\
\text { Respiratoria }\end{array}$ & 1453 & 2252 & 2371 & 2347 & 2578 & 2080 & 1663 & 2506 & 17250 \\
\hline Enf Gastroint. & 432 & 806 & 1250 & 756 & 637 & 691 & 683 & 651 & 5906 \\
\hline Gripa & 63 & 54 & 93 & 1212 & 745 & 746 & 842 & 941 & 4696 \\
\hline Tosferina & 167 & 630 & 479 & 280 & 629 & 175 & 175 & 1049 & 3584 \\
\hline Hidropesia & 261 & 246 & 262 & 279 & 258 & 263 & 211 & 160 & 1940 \\
\hline Inf. general & 274 & 278 & 248 & 301 & 229 & 204 & 187 & 190 & 1911 \\
\hline Exantémicas & 15 & 524 & 23 & 25 & 37 & 601 & 19 & 638 & 1882 \\
\hline Tifoidea & 192 & 175 & 177 & 157 & 157 & 156 & 99 & 132 & 1245 \\
\hline Anemia & 90 & 104 & 91 & 80 & 82 & 60 & 48 & 35 & 590 \\
\hline Neurológicas & 63 & 53 & 73 & 83 & 82 & 77 & 60 & 80 & 571 \\
\hline Perinatal & 69 & 47 & 28 & 60 & 82 & 50 & 87 & 72 & 495 \\
\hline Accidentes & 43 & 45 & 48 & 59 & 72 & 54 & 40 & 58 & 419 \\
\hline Cardiovascul. & 27 & 49 & 54 & 46 & 51 & 49 & 43 & 48 & 367 \\
\hline Subita & 47 & 38 & 39 & 46 & 32 & 29 & 36 & 46 & 313 \\
\hline Ahogamiento & 22 & 39 & 38 & 28 & 46 & 30 & 33 & 24 & 260 \\
\hline Renal & 13 & 20 & 29 & 31 & 28 & 32 & 24 & 30 & 207 \\
\hline Cancer-tumor & 9 & 16 & 11 & 24 & 30 & 31 & 23 & 29 & 173 \\
\hline Desnutrición & 18 & 12 & 18 & 26 & 24 & 22 & 6 & 27 & 153 \\
\hline Anemia trop. & 8 & 8 & 16 & 16 & 42 & 25 & 18 & 9 & 142 \\
\hline Violencia & 17 & 8 & 10 & 15 & 12 & 10 & 5 & 15 & 92 \\
\hline Frio-insolación & 2 & 9 & 8 & 5 & 3 & 5 & 3 & 9 & 44 \\
\hline Hemorragia & 0 & 6 & 4 & 6 & 2 & 4 & 4 & 7 & 33 \\
\hline Curiosa & 52 & 39 & 43 & 30 & 17 & 23 & 26 & 33 & 263 \\
\hline Desconocida & 367 & 308 & 206 & 159 & 134 & 161 & 147 & 200 & 1682 \\
\hline Mal definida & 95 & 132 & 115 & 111 & 104 & 84 & 74 & 85 & 800 \\
\hline Sin dato & 1960 & 1891 & 1273 & 933 & 816 & 720 & 434 & 469 & 8496 \\
\hline Total general & 5759 & 7789 & 7007 & 7115 & 6929 & 6382 & 4990 & 7543 & 53514 \\
\hline
\end{tabular}


La gripa representó un $8,8 \%$, y la tosferina un 6,7\%, del total de defunciones, sumada estas dos causas a las respiratorias, superan el $47 \%$, de la mortalidad, ubicándose entre las 5 primeras causas en los menores de 8 años. La gripa en 1918-19 como evento pandémico resulta ser un marcador de comportamiento epidemiológico pasando de una causa no presente en el periodo prepandémico de 1912-1917, a ser la segunda causa en el periodo postpandémico (1920-1927) aportando una mayor carga de mortalidad a las enfermedades respiratorias.

Aunque en el periodo analizado, las enfermedades exotérmicas se ubican en el séptimo lugar, existen olas epidémicas que las ubican en segundo o tercer lugar. Comportamiento cíclico y alternado con las cuatro primeras enfermedades infecciosas como se observa en la Figura 1.

Figura 1. Comportamiento de la mortalidad por enfermedades epidémicas en menores de 8 años, 1912-1927 en Boyacá.

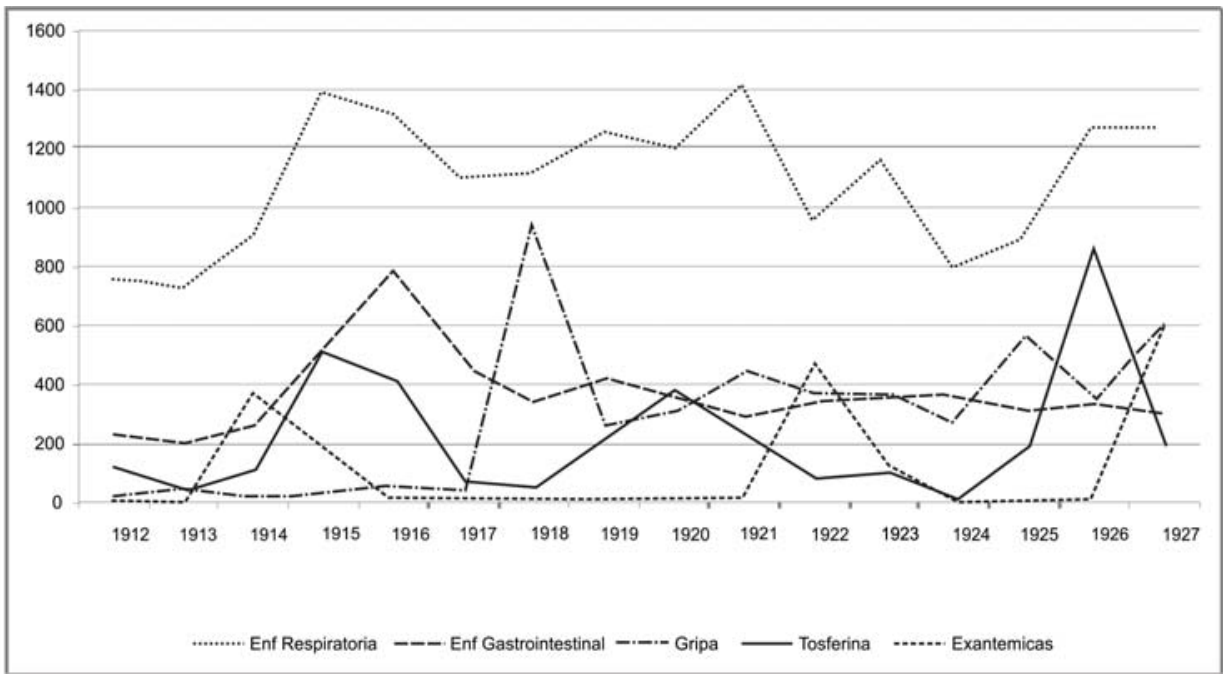

Las causas de mortalidad en el año 2005 reportadas por el DANE y la Secretaria de Salud de Boyacá, aunque a veces divergentes en otros periodos coinciden en este año en las cifras reportadas. Se observa que las enfermedades respiratorias persisten como primera causa de mortalidad, las enfermedades gastrointestinales muestran un descenso importante pasando de ser la segunda causa a comienzos del siglo XX a la sexta en el inicio del siglo XXI (Tabla 4). 
Sorprende el enorme aumento de las causas violentas y accidentales en el siglo XXI, ubicadas como tercera causa, que no aparecían dentro de las primeras 10 causas en el periodo 1912-1927. Las enfermedades congénitas que no aparecen registradas en el siglo XX aparecen como segunda causa de mortalidad en menores de 5 años en el año 2005. Las enfermedades perinatales y DNT que no estaban dentro de las 10 primeras causas en el siglo XX, se convierten en la cuarta y séptima causa en 2005.

Tabla 4. Mortalidad por causas en el 2005 en menores de 5 años en Boyacá

\begin{tabular}{lc}
\hline Causa & Muertes \\
\hline Enfermedades respiratorias & 153 \\
Malformaciones congénitas & 81 \\
Accidentalidad y violencia & 68 \\
Perinatal & 52 \\
Infección general & 30 \\
Enfermedades gastrointestinales & 19 \\
Desnutrición & 9 \\
Neurológicas & 5 \\
Linfáticas & 5 \\
Cardiovasculares & 3 \\
Renales & 2 \\
Mal definidas & 13 \\
Otras & 9 \\
Total & 449 \\
\hline Fuente: DANE 2005 adaptada por los autores
\end{tabular}

Tabla 5. Mortalidad en menores de 5 años1970-2010 por 1000 nacidos vivos en varios países

\begin{tabular}{lccccc}
\hline País & 1970 & 1980 & 1990 & 2000 & 2010 \\
\hline Colombia & 86,8 & 52,8 & 33,3 & 24,7 & 15,3 \\
Bolivia & 220,7 & 153 & 103,9 & 62,2 & 46,7 \\
Ecuador & 129,1 & 82,5 & 50,1 & 34 & 21 \\
Venezuela & 58,8 & 38,6 & 29,4 & 23,2 & 16,1 \\
Brasil & 120,8 & 83,6 & 52 & 30,8 & 19,9 \\
Argentina & 72,6 & 37,7 & 28 & 19,7 & 12,9 \\
Cuba & 38,9 & 23,3 & 13,9 & 8,6 & 5,2 \\
México & 107,5 & 70 & 41,9 & 25,8 & 16,6 \\
Canadá & 26,1 & 12,9 & 8,8 & 6,6 & 4,9 \\
E.U & 25,7 & 16 & 11,6 & 8,3 & 6,7 \\
Portugal & 74,4 & 29,4 & 15 & 8 & 3,3 \\
España & 32,8 & 14,3 & 9,2 & 5,7 & 3,8 \\
UK & 21,8 & 15,2 & 9,7 & 6,8 & 5,3 \\
Suecia & 12,7 & 8,9 & 7,2 & 4,9 & 2,7 \\
Fuente: Adaptado por los autores de Rajaratnam JK(16)
\end{tabular}

Los resultados anteriores evidencian una mejora en la atención del grupo menor de 5 años en Boyacá para el comienzo del siglo XXI, tendencia que se observaba también a comienzos del siglo XX. Contrasta que la reducción 
de 60 muertes desde 1913 a 1927 (Tabla 2) fue de 374,5 a 314 defunciones en menores de 8 años por 1000 Menores de un año, evento muy similar con cifras reportadas para Colombia, (Tabla 5) donde se redujo en 71,5 defunciones por cada 1000 nacidos vivos en Colombia en 40 años desde 1970 al 2010.

También se puede observar esta reducción en varios países de Latinoamérica y otros países con diferentes niveles de desarrollo (Tabla 5).

Mortalidad Infantil

De la misma forma que en la mortalidad bruta y la mortalidad específica en menores de 8 años, la mortalidad infantil se ve incrementada durante los años 1915, 1916, 1918 y 1921; esto es reflejo del impacto de la pandemia de gripe española en 1918-19 (Tabla 2).

En el periodo 2001 - 2005 se observa una importante reducción en la tasa de mortalidad infantil y en el número de defunciones comparada con el promedio del periodo de 1913-1927. De 2008 muertes promedio a comienzos del siglo XX se pasó a 371 muertes en promedio entre 2000 y 2005, reduciendo un $81 \%$ las defunciones infantiles y la tasa redujo notablemente de 212 a 17,4 muertes por 1000 nacidos vivos, tasa que representa una disminución del 91,8 \%, esta vez el cambio sucede tanto en valores absolutos como relativos, a pesar de que la población se duplico pasando en promedio de 9672 niños menores de 1 año entre 1913-1927 a 21316 promedio en el periodo 2000-2005.

La población a comienzo del siglo XX creció constante y escalonadamente duplicándose en el periodo estudiado, sin embargo en el siglo XXI la población se estabiliza y luego presenta tendencia a la reducción.

Más de la mitad de la mortalidad infantil (51,7 \%) en el periodo 19121927, es causada por enfermedades respiratorias que incluyen: La enfermedad respiratoria aguda con $36,5 \%$, la gripa con $8,5 \%$ y la tosferina $6,7 \%$. Esto podría ser mayor teniendo en cuenta que las causas mal definidas, sin dato y desconocidas, representan el 23,6 \% de la mortalidad infantil.

La gripa en este grupo, escasa como causa de mortalidad infantil durante el periodo 1912-1917, se vuelve una enfermedad endémica tras la pandemia de 1918-1919 pasando de no estar dentro de las diez primeras causas a ocupar el segundo o tercer lugar como causa de muerte. 
Las enfermedades gastrointestinales representan la tercera causa de mortalidad infantil con un 7,3\%, las exantémicas solo representan el 2,1\% de la mortalidad infantil registrada en el periodo. Las muertes por enfermedades inmunoprevenibles como el sarampión, agrupados en las enfermedades exantémicas aumentan las defunciones comportándose con picos epidémicos en los años de 1914-15 y 1922-23 y 1927 (Figura 2 y Tabla 6).

Proporción de mortalidad infantil

Este indicador refleja el peso que tiene la mortalidad en los grupos etáreos respecto a la mortalidad general; la mortalidad infantil representaba una cuarta parte de las muertes en promedió en el periodo 1912-1927 paso a representar un 6,7\%; caso similar en los menores de 8 años que aportaban el $42 \%$ de la mortalidad general a comienzo del siglo XX a $8 \%$ en pro medio en los menores de 5 años a comienzo del siglo XXI. Tabla 6.

Tabla 6. Proporción de mortalidad para menores de 1 año 5 años y 8 años, en Boyacá

\begin{tabular}{ccc}
\hline Año & $\begin{array}{c}\text { Proporción Mortalidad } \\
\text { Infantil en \% }\end{array}$ & $\begin{array}{c}\text { Proporción Mortalidad } \\
\text { < de 8 años en \% }\end{array}$ \\
\hline 1912 & 23,7 & 36,8 \\
1913 & 28,5 & 43,4 \\
1914 & 24,5 & 44,1 \\
1915 & 23,0 & 44,2 \\
1916 & 21,7 & 41,6 \\
1917 & 22,7 & 36,6 \\
1918 & 24,2 & 38,9 \\
1919 & 28,0 & 43,8 \\
1920 & 25,1 & 41,2 \\
1921 & 30,6 & 44,5 \\
1922 & 22,0 & 42,0 \\
1923 & 26,3 & 42,0 \\
1924 & 21,2 & 36,3 \\
1925 & 27,8 & 41,9 \\
1926 & 28,0 & 46,9 \\
1927 & 25,9 & 47,2 \\
\hline Año & Proporción Mortalidad & Proporción Mortalidad \\
\hline 2000 & Infantil en \% & de años en \% \\
2001 & 7,1 & 8,9 \\
2002 & 7,0 & 8,6 \\
2003 & 6,8 & 8,3 \\
2004 & 5,8 & 7,2 \\
2005 & 5,8 & 7,4 \\
\hline
\end{tabular}

La proporción de la población menor de 1 año se mantiene en los dos periodos de estudio en menos del $2 \%$, los menores de 8 en menos del 11 $\%$, y menores de 5 en menos de $10 \%$, respecto al total poblacional, si se 
mantiene la proporción promedio por años se esperaría que los menores de 8 en el periodo 2000-2005 sea de casi $20 \%$, duplicando la población de este grupo etáreo a comienzos del siglo XX.

\section{DISCUSIÓN}

A pesar de las dificultades en las definiciones técnicas de las tasas de mortalidad, el subregistro a principios del siglo XX, las desinformación en el siglo XXI con datos inexactos y cifras que privilegian indicadores de gasto en salud; se pudo establecer el perfil de mortalidad infantil y en menores de 8 y 5 años con respecto a la mortalidad general en dos épocas históricas durante los inicios de los siglos XX y XXI.

Un indicador negativo de la eficacia de los sistemas de registro y los métodos de clasificación de las enfermedades es el que aparezca dentro de las primeras 10 causas las mal definidas tanto a inicios del siglo XX como en el siglo XXI. La tasa de mortalidad bruta a comienzo del siglo XX, se redujo en 58,6 muertes por 10 habitantes en 16 años, reducción natural sin producirse cambios significativos en las políticas de higiene de la época.

De 1912-1927 las enfermedades infecciosas, se comportaron acorde con el patrón característico de las regiones con bajo grado de desarrollo socioeconómico (1); la pandemia de gripa de 1918-19 es un punto transicional, que cambia el grupo de afectación por mortalidad en ancianos en la prepandemia al grupo de niños en la pospandemia (13).

Para los años 1915 y 1916 los informes del gobernador de Boyacá a la asamblea departamental consignan epidemias de viruela y tifo y en 1921 de viruela, fiebre tifoidea, gripa, tuberculosis, sífilis y difteria en Boyacá (13). La reducción observada de las tasas de mortalidad brutas de inicios del siglo XXI comparada con la registrada a comienzo del siglo XX, se explica por el establecimiento de un Ministerio de Salud que comienza su actuar en la segunda mitad del siglo XX y un sistema de salud organizado en 1979 y reorganizado en 1993.

Los cambios en la mortalidad en menores de 8 años de 1915-16 se relacionan posiblemente con las epidemias de disentería, tos ferina, difteria y viruela, registradas en los informes del gobernador de Boyacá a la 
asamblea departamental de la época $(13,14)$. En la mortalidad de menores de 8 años se registra un ascenso leve en 1914, cuando los informes del gobernador a la Asamblea notifican epidemias de "viruela" y "tifo" en Boyacá, enfermedades con alta mortalidad en este grupo.

En estos grupos poblacionales se observa una efectividad de la política sanitaria reflejada en el descenso absoluto de las defunciones, pasando 3 843 muertes en 1927 a 449 en el año 2005. Hay que considerar que en el primer grupo el intervalo es de 8 años mientras que en el segundo es de 5 años esto por los ajustes en la definición de los rangos para los censos y la notificación en las instituciones de salud.

A comienzo del siglo XX, la única vacuna aplicada era contra la viruela, solo en momentos de epidemias y no como un programa regular. En el siglo XXI existe un Programa Ampliado de Inmunización (PAI) con gran cobertura que redujo considerablemente este tipo de enfermedades y su mortalidad en los menores de 5 años.

En la pre pandemia la mortalidad general registro oscilaciones marcadas que se corresponden con la aparición de brotes de enfermedades infecciosas reportadas en la época como disentería, tifo, fiebre tifoidea y anemia tropical, entre otras. La Junta Departamental de Higiene informa "endemias mortíferas" de anemia tropical, pián, paludismo, lepra, carate y tuberculosis, en 1914. En Occidente predomina el paludismo y las bautizadas por Roberto Franco, como Fiebres de Muzo (12,13). En 1916, se insiste en que: "uno de los males que más mortalidad produce en climas templados es la anemia tropical" y, en 1919, se afirma que "la lepra, la tuberculosis, la sífilis y el alcoholismo diezman nuestras poblaciones" $(13,14)$. Las tierras bajas reportan tifo, paludismo, disentería y anemia tropical. En 1917, se ordena que en todos los municipios de Boyacá debe crearse una Junta de Sanidad "compuesta de dos vecinos honorables" que residan en la población, nombrados por los concejos $(12,13)$.

Los hallazgos epidemiológicos encontrados en la base de datos que soportan la existencia de estas epidemias ameritan un análisis que se abordará en el futuro.

El indicador de proporción de mortalidad refleja la prioridad de la política de infancia, disminuyendo la mortalidad absoluta en menores de 
1 y 5 años frente a la mortalidad en la población general. Este cambio reduce la mortalidad por enfermedades infectocontagiosas pero mantiene en aumento las causas externas como accidentalidad y violencia tornándose como preocupación actual sin evidencia de políticas específicas de control. Indicador sensible al solo hecho de muerte sin considerar las políticas de planificación familiar vigentes en el siglo XXI.

Además del indicador definido por la OMS, denominado Mortalidad en menores de 5 años respecto a mil NV,(6) se debería considerar como indicador de trazador de políticas la tasa de mortalidad específica en menores de 5 años ya que esta refleja realmente el problema en el grupo en mención mientras que la de la OMS es una razón y que compara dos grupos poblacionales diferentes.

La mortalidad en menores de 5 años por mil NV, es un indicador de desarrollo de los países que va en descenso a nivel global como resultado de las políticas internacionales lideradas por la OMS y la UNICEF. Su tendencia en el tiempo difiere entre países desarrollados, en vías de desarrollo y no desarrollados la tendencia en todos es hacia la disminución. Colombia se encuentra en un estadio intermedio superada por Cuba y los países del cono sur (16), Boyacá se encuentra con cifras ligeramente superiores al promedio nacional.

Las autoridades sanitarias deberán unificar los sistemas de información de manera que permitan a los trabajadores de la salud y a los gestores de políticas en salud tener indicadores fiables, oportunos y no solo enfocados hacia el gasto en salud. Lo anterior respecto al caso del boletín epidemiológico de Boyacá del 2007 en el que la mayoría de los indicadores están calculados para el grupo de los no asegurados fuera del régimen contributivo y subsidiado

Agradecimientos: A los estudiantes de Medicina de la UPTC por el trabajo de campo en la sistematización de los registros de defunción parroquiales del Departamento de Boyacá. 


\section{REFERENCIAS}

1. Dever G. Epidemiologia y Administración de Servicios de Salud. Washington D.C.: Organización Panamericana de la Salud; 1991.

2. Valenzuela B, Oryan G. Logros y desafíos del Programa Ampliado de Inmunizaciones en la región de las Américas. Rev méd Chile 2000; 128(8):911-922.

3. Romero M, Bedregal P, Bastías G. Situación de la Salud Materno Infantil en Chile. Boletín Salud Pública. [Internet]. Disponible en: http://escuela.med.puc.cl/publ/Boletin/ SaludPublica/ SituacionSalud.html. Consultado Mayo 2010.

4. Secretaria de Salud. Mortalidad preescolar. Boletín Hospital Infantil México 2005; 62: 69-82.

5. DeCS Server-List Terms [Internet]. Disponible en: http://decs.bvs.br/cgibin/ wxis1660.exe/ decsserver/. Consultado Mayo 2010.

6. OMS. La contribución para el logro de las Metas de Desarrollo de la Declaración del Milenio de Naciones Unidas. Informe del Secretariado; 2003.

7. Manrique-Abril F. Epidemiología social y salud Pública. Tunja; Universidad Pedagógica y Tecnológica de Colombia; 2003.

8. Martínez-Martín A. El Lazareto de Boyacá: Lepra, medicina, iglesia, y Estado 18691916: cómo Colombia fue convertida en la primera potencia leprosa del mundo, y Boyacá, en una inmensa leprosería. Tunja; Universidad Pedagógica y Tecnológica de Colombia; 2006.

9. Peñuela V. Apuntaciones de geografía e historia médicas del departamento de Boyacá en la República de Colombia. Revista Médica de Bogotá 1924; 487-498:64-141.

10. Quevedo V. Historia de la medicina en Colombia. Hacia una profesión liberal, 1865-1918. Cali: Tecnoquímicas-Grupo Editorial Norma; 2009.

11. DANE. Censo general 2005: Nivel nacional. Bogotá; DANE Departamento Administrativo Nacional de Estadística; 2008.

12. Manrique FG, Martínez AF, Ospina JM, Meléndez B. La pandemia de gripe de 1918-1919 en Bogotá y Boyacá, 91 años después; Infectio. 2009; 13(3):182-191.

13. Martínez A, Ospina JM, Meléndez BF, Manrique-Abril F. Antes, durante y después de la visita de la "Dama Española". Varia hist. 2009; 25(42):499-517.

14. Ospina JM, Martínez AF, Herrán O. Impacto de la pandemia de gripa de 1918-1919 sobre el perfil de mortalidad general en Boyacá, Colombia. Hist cienc saude-Manguinhos. 2009; 16(1): 345-67.

15. Rodríguez J, Ruiz F, Peñaloza E, Eslava J, Gómez L, Sánchez H. Encuesta Nacional de Salud 2007.Departamento de Boyacá. Bogotá: Javegraf; 2009.

16. Rajaratnam JK, Marcus JR, Flaxman AD, Wang H, Levin-Rector A, Dwyer L, et al. Neonatal, postneonatal, childhood, and under-5 mortality for 187 countries,1970-2010: a systematic analysis of progress towards Millennium Development Goal 4. The Lancet. 2010; 375(9730):1988-2008. 